\title{
Educação em saúde sobre a dengue em uma escola: Relato de experiência
}

\section{Health education about dengue in a school: An experience report \\ Educación sanitaria sobre el dengue en una escuela: Un relato de experiencia}

Luana Gomes de Lima Martins ORCID: https://orcid.org/0000-0002-8076-0397 Universidade do Estado do Pará, Brasil

E-mail: luanagomesdelima7@gmail.com

Ingrid Magali de Souza Pimentel ORCID: https://orcid.org/0000-0003-1820-5496 Universidade do Estado do Pará, Brasi E-mail: imbarleta@yahoo.com.br

Tatiana Menezes Noronha Panzetti ORCID: https://orcid.org/0000-0001-7560-4595 Universidade do Estado do Pará, Brasil E-mail: tnpanzetti@hotmail.com

Ilma Pastana Ferreira

ORCID: https://orcid.org/0000-0002-9152-3872 Universidade do Estado do Pará, Brasil E-mail: pastanailma@gmail.com

Renata de Jesus da Silva Negrão ORCID: https://orcid.org/0000-0003-0364-0700 Centro Universitário FIBRA, Brasil

E-mail: renatanegrao@outlook.com.br Margarete Feio Boulhosa

ORCID: https://orcid.org/0000-0003-1680-9368 Universidade do Estado do Pará, Brasil E-mail: margareteboulhosa@gmail.com

Dione Seabra de Carvalho

ORCID: https://orcid.org/0000-0001-5342-6820 Faculdade Cosmopolita, Brasil

E-mail: diseabra@bol.com.br

Nathália Menezes Dias

ORCID: https://orcid.org/0000-0002-2166-5293 Universidade do Estado do Pará, Brasil

E-mail: menezesdiasnathalia@gmail.com

Luanda da Silva e Silva

ORCID: https://orcid.org/0000-0001-7097-3068 Universidade do Estado do Pará, Brasil E-mail: luandacat.crazy@gmail.com

Cristalia de Melo da Silva

ORCID: https://orcid.org/0000-0002-2449-387X Universidade do Estado do Pará, Brasil

E-mail: cristaliademelo@gmail.com

Ana Caroline de Oliveira Coutinho ORCID: https://orcid.org/0000-0003-2558-9643 Secretaria Municipal de Saúde de Tucuruí, Brasil E-mail: coutinhoanacaroline@gmail.com Fabiane dos Santos Ferreira

ORCID: https://orcid.org/0000-0003-4484-8233

Centro Universitário FIBRA, Brasil E-mail: fabianedsferreira@gmail.com

Atilio Rodrigues Brito

ORCID: https://orcid.org/0000-0003-2180-6057 Universidade do Estado do Pará, Brasil

E-mail: atiliorodrigues2010@ hotmail.com

João Victor Corrêa Pinto

ORCID: https://orcid.org/0000-0003-2513-4657 Universidade Federal do Pará, Brasil E-mail: JoaoVictorCorrea19990408Pinto@gmail.com

Michelle Quaresma Cardoso

ORCID: https://orcid.org/0000-0002-8621-8670 Universidade do Estado do Pará, Brasil E-mail: michelle.card91@yahoo.com.br 


\begin{abstract}
Resumo
Relatar a experiência acadêmica de uma educação em saúde sobre a dengue realizada em uma escola Estadual no município de Belém-PA. Trata-se de um estudo qualitativo e descritivo, do tipo relato de experiência, relatando a educação em saúde sobre a dengue, desenvolvida no interior de uma escola Estadual no município de Belém-PA, com a participação de alunos do $6^{\circ}$ ano, realizada por uma equipe multiprofissional de graduandos. A ação educativa se dividiu em basicamente dois momentos, o primeiro foi o que trouxe aos alunos uma abordagem mais teórica sobre a dengue, e o outro se caracterizou como um momento mais prático. No primeiro momento e dia da ação os alunos puderam sanar suas dúvidas e contaram suas vivências e fatos em relação a dengue após a explanação do assunto. Os alunos também fizeram uma produção do que foi exposto pelos graduandos e nessa produção, foi perceptível observar a criatividade e aprendizagem dos alunos enquanto ao assunto abordado, pois nos desenhos, pinturas, cartazes feitos por eles tinham aspectos da dengue que foram relatados previamente como, por exemplo, o mosquito, onde eles podem se reproduzir e outros. Além disso houve o momento mais prático da ação, onde os alunos paramentados com EPIs foram em busca dos possíveis focos dos mosquitos dentro do ambiente escolar. Conclui-se então que um olhar integral da metodologia expôs que os alunos irão atuar como pontarias de dissipação de medidas preventivas contra a dengue.
\end{abstract}

Palavras-chave: Dengue; Promoção da saúde no ambiente escolar; Alunos.

\begin{abstract}
To report the academic experience of a health education about dengue held in a state school in the city of Belém-PA. This is a qualitative and descriptive study, of the experience report type, reporting health education about dengue, developed in a state school in the city of Belém-PA, with the participation of 6th grade students, carried out by a multiprofessional team of undergraduates. The educational action was basically divided into two moments, the first was the one that brought students a more theoretical approach to dengue, and the other was characterized as a more practical moment. In the first moment and day of the action, the students were able to resolve their doubts and told their experiences and facts in relation to dengue after explaining the matter. The students also made a production of what was exposed by the undergraduates and in this production, it was noticeable to observe the creativity and learning of the students regarding the subject covered, because in the drawings, paintings, posters they made had aspects of dengue that were previously reported as, for example, the mosquito, where they can breed and others. In addition, there was the most practical moment of the action, where students dressed with PPE went in search of possible outbreaks of mosquitoes within the school environment. It is concluded then that a comprehensive look at the methodology showed that students will act as aims to dissipate preventive measures against dengue.
\end{abstract}

Keywords: Dengue; Health promotion in the school environment; Students.

\title{
Resumen
}

Informar la experiencia académica de una educación para la salud sobre el dengue realizada en una escuela pública de la ciudad de Belém-PA. Se trata de un estudio cualitativo y descriptivo, del tipo relato de experiencia, informando la educación en salud sobre el dengue, desarrollado dentro de una escuela pública en la ciudad de Belém-PA, con la participación de estudiantes de $6^{\circ}$ grado, realizado por un equipo multiprofesional de pregrado. La acción educativa se dividió básicamente en dos momentos, el primero fue el que trajo a los estudiantes un acercamiento más teórico sobre el dengue, y el otro se caracterizó por ser un momento más práctico. En el primer momento y día de la acción, los estudiantes pudieron resolver sus dudas y contaron sus experiencias y hechos en relación al dengue luego de explicar el asunto. Los estudiantes también hicieron una producción de lo expuesto por los estudiantes de pregrado y en esta producción, se notó observar la creatividad y el aprendizaje de los estudiantes con respecto al tema tratado, pues en los dibujos, pinturas, carteles que hicieron tenían aspectos del dengue que Se informaron anteriormente como, por ejemplo, el mosquito, donde pueden reproducirse y otros. Además, se produjo el momento más práctico de la acción, donde los alumnos con EPP acudieron en busca de posibles brotes de mosquitos dentro del entorno escolar. Se concluye entonces que una mirada integral a la metodología mostró que los estudiantes actuarán como objetivos para disipar las medidas preventivas contra el dengue.

Palabras clave: Dengue; Promoción de la salud en el entorno escolar; Estudiantes.

\section{Introdução}

O vírus da dengue, apesar de não ser nativo das Américas, adentrou no Brasil a partir da África, possivelmente no início do século XIX, onde encontrou um ambiente propiciou para sua procriação e desenvolvimento, o que promoveu sua disseminação territorial em terras brasileiras. Apesar de ser uma patologia provecta, a representação de morbimortalidade e a necessidade de variadas táticas para sua restrição, geram uma aproximação de cerca de 100 milhões casos ao redor do mundo. Dessa maneira, as epidemias contínuas nos últimos 20 anos no país, colocaram a dengue fundamento de debate nas vias de 
unidades de saúde, escolas, comunicação, em todas as instâncias sociais, além de extensas campanhas promovidas pelo Ministério da Saúde, Secretarias Estaduais e Municipais de Saúde (Medeiros et al., 2013; Vasconcelos, 2015).

A dengue no Brasil se caracteriza como uma das mais importantes e recorrentes arboviroses no ser humano, ocasionando um grande problema de saúde pública no país. Isso devido à sua alta adaptação ao clima brasileiro, tropical, adequando-se assim, e proporcionando uma maior disseminação de criadouros do seu principal vetor, o Aedes Aegypti. Tal doença apresenta-se como uma doença febril aguda e possui formas branda e grave da doença, sendo dissociada de acordo como a doença se apresenta, podendo ser disposta de infecção inaparente, dengue clássico (DC), febre hemorrágica da dengue (FHD) ou síndrome do choque da dengue (SCD) (Brasil, 2005; Chaves, Evangelista \& Fernandes, 2020).

Segundo Lopes, Nozawa e Linhares (2014) para todos os casos, a dengue pode ter manifestação oligo ou assintomática, podendo ocorrer hemorragias ou choques, podendo levar ao óbito, se caracterizando por febre alta abrupta seguida de cefaléia, mialgia, prostração, artralgia, anorexia, astenia, dor retroorbital, náuseas, vômitos, exantema, prurido cutâneo .Além disso, tal mazela se torna constante devido aos focos de criadouros de seus vetores, se relacionando assim com as más condições de saneamento básico da localidade (Brasil, 2005).

Sendo assim, a educação popular em saúde torna-se uma grande arma de combate a essa mazela social, partindo-se do pressuposto que tal dinâmica visa a mudança de pensamento no agir da população para que haja nela cada vez mais, um agir crítico, integral e humanizado, promovendo a emancipação e a construção de um pensamento voltado ao aspecto do bem coletivo, sendo assim, tal prática torna-se indispensável no combate à dengue, no que diz respeito a diminuição dos focos de criadouros do vetor da doença por parte da comunidade (Botelho, Cruz, Bornstein, David, Scherlowski \& Lima, 2021).

Diante de tal ocorrência, a abordagem da educação popular em saúde se torna uma grande estratégia, principalmente, se abordado para a população jovem da comunidade (crianças e jovens), tendo em vista que o quanto antes a formação de conhecimento for prestada a comunidade, o pensamento crítico-reflexivo se torna mais fortemente enraizado dentro da sociedade, facilitando processos de mudança de comportamento social, além de mobilização e processos sociais no campo da saúde (Botelho et al., 2021).

Para além, tal estratégia de combate à dengue se torna ainda mais eficaz, quando feito perante uma abordagem multiprofissional, pois tal abordagem apresenta como objetivo o aumento do acesso da população as orientações e conhecimentos em saúde, possibilitando uma interação entre disciplinas e profissões tidas como indispensáveis para a abordagem do processo de saúde-doença dentro da comunidade assim como uma maior interação dentro da mesma (Barreto, Rebouças, Aguiar, Barbosa, Rocha, Cordeiro, Melo \& Freitas, 2019)

Diante do exposto, o estudo objetivou relatar a experiência acadêmica de uma educação em saúde sobre a dengue, realizada em uma escola Estadual no município de Belém-PA.

\section{Metodologia}

Trata-se de um estudo qualitativo e descritivo, do tipo relato de experiência, relatando a educação em saúde sobre a dengue, que foi desenvolvido no interior de uma escola Estadual no município de Belém-PA, com a participação de alunos do $6^{\circ}$ ano do ensino fundamental, realizado por uma equipe multiprofissional de graduandos de uma Universidade.

O estudo que se utiliza de uma abordagem qualitativa se refere àquele que apresenta a apreciação do pesquisador, trazendo consigo os subsídios do mesmo sobre a problemática do estudo. Relacionado a isso, esse tipo de pesquisa expõe algumas particularidades como, por exemplo, o investigador é de fundamental significância dentro dessa metodologia; a coleta de informações são preferencialmente descritivas; as concepções que as pessoas dão as coisas e a tudo ao seu redor são de extrema relevância para o investigador; a inquietude da pesquisa se sobrepõe sobre o resultado e; as informações e 
ponderações dos dados alcançados inclinam-se a conduzir um processo indutivo (Martins et al., 2021).

As pesquisas descritivas apresentam como finalidade primordial a descrição das propriedades de determinada comunidade ou episódio, ou a instalação de semelhanças entre variáveis. São numerosos os estudos que podem ser rotulados sob este título e uma de suas peculiaridades mais importantes surge do uso de métodos padronizadas de coleta de dados. Esse tipo de estudo, busca descrever uma situação ou fenômeno minuciosamente, sobretudo o que está acontecendo, consentindo envolver, com exatidão, as propriedades de um indivíduo, grupo ou uma situação, bem como expuser a relação entre os acontecimentos (Oliveira, 2011; Tabosa, Pinto \& Loureiro, 2016).

O relato de experiência está além de uma descrição sucinta sobre algum fato ou evento, pois ao realizar a sua leitura, é verossímil compreender com mais fidedignidade a experiência abordada. Além do mais, propicia teoricamente equipará-la com outas vivências similares, possibilitando um melhor feedback sobre a temática abordada (OIiveira, Maia, Panzetti, Souza \& Galvão, 2020).

A ação se dividiu em basicamente em dois momentos, do qual o primeiro foi o que trouxe aos alunos uma abordagem mais teórica sobre a dengue, e o outro se caracterizou como um momento mais prático, possibilitando aos alunos identificarem dentro do ambiente escolar os focos de dengue, a sua eliminação e os métodos de prevenção, para assim atuarem como influenciadores e interventores dentro problemática em todos os ambientes, inclusive em seus domicílios.

No dia da ação os responsáveis por tal experiência, pautada na realização de uma ação educativa, se apresentaram como graduandos de cursos da área da saúde de uma Universidade. Em seguida os mesmos fizeram uma abordagem teórica com relação à temática abordada, e para isso utilizaram cartazes, considerada uma tecnologia leve, para mostrar aos estudantes considerações como, por exemplo, uma visão geral sobre a dengue, meios de transmissão, prevenção e prováveis medidas de controle, assim como sua relação com o meio ambiente (Pereira \& Lemes, 2018).

Após a exposição do assunto, a turma foi separada em duas equipes e acenada a mostrar o que foi apreendido e o que mais lhes chamou a atenção; esse processo se deu por meio da produção de textos, cartazes e imagens s que demostravam o que é dengue e tudo que está ligada a ela. Toda produção feita pelos alunos foi em seguida colocada em lugares estratégicos dentro do ambiente escolar, para que assim todos os demais alunos fossem alcançados e pudessem também apreender um pouco da temática em questão.

Os alunos também puderam sanar suas dúvidas por meio de um diálogo informal e perguntas dirigidas. Essa proposta se faz pertinente, uma vez que, a prevenção e controle dessa patologia se dão pela participação da comunidade, por intermédio de atividades educacionais como esta, a fim de conscientizar a população (Santana \& Costa, 2016).

Em consonância a isso e através de um diálogo, os alunos foram informados que os acadêmicos retornariam no dia seguinte para darem prosseguimento das atividades desse segundo momento. Esse segundo dia de continuidade da ação, os graduandos anunciaram sobre as atividades a serem procedidas e todos os cuidados necessários que precisavam ter para $o$ cumprimento das mesmas como, por exemplo, o uso de equipamentos de proteção individual (EPIs) indispensáveis como luvas e calçados fechados, para então dá início ao reconhecimento e eliminação de prováveis focos do vetor da dengue (Brasil, 2019).

O segundo momento representativo da ação foi mais prático e apresentou como objetivo mostrar aos alunos as ações e intervenções que devem e podem ser seguidas para impedir a aparição e proliferação do mosquito da dengue e por conseguinte da doença, mostrando aos mesmos como agir e que medidas devem ser adotadas. Desta forma, o ambiente utilizado para esse momento foi a área da escola e suas adjacências. 


\section{Resultados e Discussão}

Para Leonello e L'Abbate (2006) quando se houve falar em promoção à saúde pensa-se na constituição de práticas que contribuem para construção e promoção de praxes saudáveis, pessoas autônomas, responsáveis e que reconhecem seus direitos relacionado à saúde. Para a evolução de tais práticas, se faz essencial ponderar a imprescindibilidade de delinear campos de ação para a promoção da saúde. Dentre as quais se apresentam: a constituição de ambientes favoráveis; a construção de políticas públicas saudáveis; o desenvolvimento de aptidões individuais; reorientações dos serviços de saúde; e o apoio da ação comunitária, por meio do compromisso social.

Uma das estratégias que promovem a responsabilidade social é a Educação em Saúde, que é compreendida como a associação de atitudes e experiências de aprendizado com intuito de promover o entendimento das pessoas sobre os determinantes da saúde, sobre condições socioeconômicas que impactam na saúde do individual e coletividade, e sobre o comportamento em saúde (Leonello, \& L'Abbate, 2006).

Relacionado a isso, realizou-se a educação em saúde e no primeiro momento e dia da ação onde houve uma abordagem teórica sobre a dengue, os alunos puderam sanar suas dúvidas e contaram suas vivências e fatos em relação a essa problemática após a explanação do assunto, o que promoveu um ambiente bastante favorável para a troca de experiências e informações enquanto temática abordada (Andrade, Prado, Albarado, Sousa \& Mendonça, 2020). Fato esse, que se assemelhou ao estudo de Barros e Neiva (2019), uma vez que, o estudo trata-se de recurso em que a compreensão científica alcança a coletividade, ofertando arcabouços para a adesão de novos comportamentos em saúde, importante no trabalho do cuidado multiprofissional, constituindo-se por meio da participação da população com o compartilhamento de suas crenças, necessidades, vivencias e cultura.

Aliado a isso e correspondente ao primeiro momento da ação, os mesmos realizaram uma atividade produtiva, na qual eles criaram conteúdos de acordo com o que apreendido durante as falas dos graduandos. Nessa produção, foi perceptível observar a criatividade e aprendizagem dos alunos enquanto ao assunto abordado, pois nos desenhos, pinturas, cartazes feitos por eles tinham aspectos da dengue que foram relatados previamente como, por exemplo, o mosquito, onde eles podem se reproduzir e outros. Dentro dessa perspectiva Vassimon (2013) possibilita observamos que, para que consigamos, de fato, raciocinar em ações na escola, e para a escola, é de fundamental importância transfigurar a criança e o adolescente em agentes da realidade, conferindo-os da relevância que lhes compete no processo do ensino e da aprendizagem.

Após a produção dos cartazes, desenhos e outros, tais foram colocados na área externa da escola para que assim também abrangesse outros alunos que não participaram diretamente da educação em saúde. Durante a colagem desses em locais estratégicos da escola como refeitório, entrada e corredor, foi visível o entusiasmo e reconhecimento dos mesmos em relação ao papel transformador que exercem dentro de um ambiente. A produção dos alunos apresentou um misto de cores alegres, o que promoveu atenção ainda maior de outros alunos que passam pelos ambientes em que estavam os mesmos (Almeida, 2018).

No segundo dia e momento da ação educativa, que correspondeu o momento mais prático da ação, em relação as medidas de eliminação e preventivas da proliferação do mosquito e consequentemente da dengue, os alunos paramentados com EPIs foram em busca dos possíveis focos dos mosquitos dentro do ambiente escolar. Nesse momento os protagonistas foram os alunos, onde analisaram, identificaram e recolheram objetos e recipientes que eram propícios para proliferação do mosquito, dentre os quais alguns objetos dispostos em alguns locais da escola já apresentavam larvas do mosquito, dessa forma agiram de maneira segura e possibilitando a eliminação e prevenção da proliferação do problema em questão (Szukala, 2010).

Por fim, todo o material apanhado pela coleta obteve a certa destinação, do qual o lixo foi reparado e encaminhado para reciclagem. Este episódio prático também procurou aproximar os alunos do problema trabalhado e também teve o intuito 
de torná-los interventores, pois a partir do conhecimento adquirido, eles tiveram/terão a capacidade de identificar e comunicar diretamente ou com a ajuda de seus familiares aos órgãos responsáveis, sobre possíveis focos da doença.

\section{Conclusão}

Conclui-se então que um olhar integral da metodologia expôs que os alunos irão atuar como pontarias de dissipação de medidas preventivas contra a dengue, e se este processo continuar a ser executado por professores e outros setores, principalmente o da saúde, teremos um quadro onde a maioria da população terá sido conscientizada quanto a este grave problema (Pereira, Rivera, \& Artmann, 2013).

Além do mais, foi possível verificar a capacidade de compreensão e interesse dos alunos a partir, principalmente, da atividade prática realizada, possibilitando analisar o quanto uma abordagem mais prática dos assuntos torna mais fácil o entendimento das pessoas. Em consonância ao supracitado, sugere-se aos estudos futuros uma abordagem mais prática da educação em saúde, a exemplo a utilizada no estudo em questão, voltada ao combate à dengue, para que assim traga à tona estudos que mostrem a eficácia e bom resultado na realização dessas atividades.

\section{Referências}

Almeida, I. M. (2018). Cores e encantos: as artes visuais na creche municipal Mercês do Amaral, na cidade de Nova Palmeira-PB. Trabalho de Conclusão de Curso (TCC) - Universidade Estadual de Paraíba. p.33.

Andrade, N. F., Prado, E. A. J., Albarado, Á. J., Sousa, M. F., \& Mendonça, A. V. M. (2020). Saúde Debate. Rio de Janeiro, 44(126), 871-880. 10.1590/01031104202012621.

Barreto, A. C. O., Rebouças, C. B. A., Aguiar, M. I. F., Barbosa, R. B., Rocha, S. R., Cordeiro, L. M., Melo, K. M., \& Freitas, R. W. J. F. (2019). Percepção da equipe multiprofissional da Atenção Primária sobre educação em saúde. Revista Brasileira de Enfermagem, 72(Suppl. 1), 266273. https://doi.org/10.1590/0034-7167-2017-0702.

Barros, K. B. C., \& Neiva, F. R. (2019). Educação em saúde como instrumento na prevenção de esquistossomose em idosos vulneráveis: Relato de experiência. In: I Congresso Paraense de Enfermagem/ $80^{\mathrm{a}}$ Semana Brasileira de Enfermagem/ I Seminário Estadual em Atenção Primária à Saúde (I COPENF/ 80ª SBEn / I SEAPS) - ABEn PA - Belém - Pará.

Botelho, B. O., Cruz, P. J. S. C., Bornstein, V., J., David, H. M., Scherlowski, L., \& Lima, L. O. (2021). Experiências de formação no contexto da Política Nacional de Educação Popular em Saúde no Sistema Único de Saúde. Interface - Comunicação, Saúde, Educação, 25, e200195. https://dx.doi.org/10.1590/interface.200195.

Chaves, M. de O., Evangelista, M. do S. N., \& Fernandes, F. M. de C. (2020). Educação em saúde sobre o Aedes aegypti: relato de experiência. Revista Brasileira de Enfermagem, 73(3), e20180487.

Leonello, V. M., \& L'Abbate, S. (2006). Educação em saúde na escola: uma abordagem do currículo e da percepção de alunos de graduação em pedagogia. Interface - Comunicação, Saúde, Educação, 10(19), 149-166. https://doi.org/10.1590/S1414-32832006000100011.

Lopes, N., Nozawa, C., \& Linhares, R. E. C. (2014). Características gerais e epidemiologia dos arbovírus emergentes no Brasil. Revista Pan-Amazônica de Saúde, 5(3), 55-64. Pereira, C. M. \& Lemes, J. R. A. (2018). Medidas de educação e saúde na escola. Em Extensão, 17(1), 191-205.

Martins, L. G. de L., Mourão, K. Q., Pimentel, I. M. de S., Panzetti, T. M. N., Ferreira, I. P., Coelho, L. A. C., Boulhosa , M. F., Carvalho, D. S. de., Gomes, V. R., Cordovil, A. B. C., Silva , L. da S. E., Ribeiro, G. M., Mata, M. G. da., Tavares, I. I. S., Oliveira, R. B. S. de., Silva, C. de M. da., Silva, L. C. M. da., Ferreira, F. dos S., Silva, F. M. da., \& Pinto, J. V. C. (2021). Nursing care for a patient with non-organic and non-specific psychosis: report of academic experience. Research, Society and Development, 10(2), e8810212274. https://doi.org/10.33448/rsd-v10i2.12274.

Medeiros, I. S., Silva, I. T. F., Silva, P. H. A., Brandão, P. S., Andrade, K. G., Lima, M., \& Melo, K. D. F. (2013). Avaliação de uma intervenção educativa na prevenção da dengue. Universidade Federal de Paraíba. Centro de Ciências Aplicadas e Educação/ Departamento de Engenharia e Meio Ambiente/ PROBEX. http://www.prac.ufpb.br/enex/trabalhos/6CCAEDEMAPROBEX2012188.pdf.

Ministério da Saúde; Secretaria de Vigilância em Saúde; Departamento de Vigilância Epidemiológica. (2005). Guia de Vigilância Epidemiológica. (6 edição).

Ministério da Saúde. (2019). Secretaria de Vigilância em Saúde. Departamento de Saúde Ambiental, do Trabalhador e Vigilância das Emergências em Saúde Pública. Manual sobre Medidas de Proteção à Saúde dos Agentes de Combate às Endemias. Arboviroses Transmitidas pelo Aedes aegypti. Brasília. 1, (1ª ed), p. 92.

Oliveira, L. F. de., Maia, C. L. do C., Panzetti, T. M. N., Souza, M. W. O. de., \& Galvão, M. M. (2020). The contribution of academic Nursing monitoring in clinical surgery on the student-monitor perspective. Research, Society and Development, 9(9), e489997374.

Oliveira, M. F. de. (2011). Metodologia científica: um manual para a realização de pesquisas em Administração. Manual (pós-graduação) - Universidade Federal de Goiás. Bibliografia. Catalão: UFG, 72 p. 
Research, Society and Development, v. 10, n. 7, e36510716291, 2021

(CC BY 4.0) | ISSN 2525-3409 | DOI: http://dx.doi.org/10.33448/rsd-v10i7.16291

Pereira, R. C. A., Rivera, F. J. U., \& Artmann, E. (2013). O trabalho multiprofissional na estratégia saúde da família: estudo sobre modalidades de equipes. Interface - Comunicação, Saúde, Educação, 17(45), 327-340.

Pereira, C. M., \& Lemes, J. R. A. (2018). Medidas de educação e saúde na escola. Revista Em Extensão, 17(1), 191-205. https://doi.org/10.14393/REEv17n12018-rel08

Santana, D. P. de., \& Costa, C. R. B. (2016). Educação Física escolar na promoção da Saúde. Revista Científica Multidisciplinar Núcleo Do Conhecimento. Edição 01, Vol. 10, 171-185. ISSN: 2448-0959.

Szukala, C. M. (2010). Representação social de saúde, doença e dengue para alunos do ensino. Dissertação (Mestrado em Psicologia) - Universidade Católica Dom Bosco, Campo Grande, p 102.

Tabosa, H. R., Pinto, V. B., \& Loureiro, J. M. M. (2016). Análise de regularidades metodológicas em pesquisas brasileiras sobre comportamentos de uso e usuários da informação. Investigación bibliotecológica, 30(70), 249-267.

Vasconcelos, P. F.C. (2015). Doença pelo vírus Zika: um novo problema emergente nas Américas? Revista Pan-Amazônica de Saúde, 6(2), 9-10.

Vassimon, G. (2013). Escuta dos aprendizes por meio dos desenhos. Construção psicopedagógica, 21(22), 132-140. 